\title{
La légende de Raphaël. Les " grandes " et les " petites " vies d'une figure exemplaire de l'art, écrites en France au cours du XIXe siècle
}

\author{
Nicole Dubreuil-Blondin
}

Volume 22, numéro 1-2, 1995

URI : https://id.erudit.org/iderudit/1072515ar

DOI : https://doi.org/10.7202/1072515ar

\section{Aller au sommaire du numéro}

\section{Éditeur(s)}

UAAC-AAUC (University Art Association of Canada | Association d'art des universités du Canada)

\section{ISSN}

0315-9906 (imprimé)

1918-4778 (numérique)

Découvrir la revue

Citer cet article

Dubreuil-Blondin, N. (1995). La légende de Raphaël. Les « grandes » et les " petites » vies d'une figure exemplaire de l'art, écrites en France au cours du XIXe siècle. RACAR : Revue d'art canadienne / Canadian Art Review, 22(1-2), 80-86. https://doi.org/10.7202/1072515ar

\section{Résumé de l'article}

Considering the lives of Raphael written in nineteenth-century France, this article examines the epistemological status and the ideological function of an important genre of art historical writing: the artist monograph. During a period characterized by new scientific ambitions and by the development of the discipline within academic institutions, the Vasarian model of the vita undergoes a number of changes that do not, however, alter in a significant way its celebrational purpose. Especially throughout the biographical anecdotes that sustain the narrative of his famous career (like the episode of his love affair with la Fornarina), Raphael remains both the great man and the angelic figure concocted by tradition, combining pagan and Christian standards of excellence, a pattern that finds itself repeated in the more popular versions of Raphael's lives, whether written for educational or for recreational purposes. The success story proposed by the life of Raphael - one of the artists most written about in France between 1800 and 1900 - establishes a poignant contrast with the destiny of the modern artist elaborated in contemporary literature, under the aegis of bohemia. It suggests that art history likes winners and not losers and that the genre of the artist monograph always serves some ideological purpose, like compensating fantasmatically for the economic, social and political insecurities of the times.
Tous droits réservés ( $)$ UAAC-AAUC (University Art Association of Canada | Association d'art des universités du Canada), 1997
Ce document est protégé par la loi sur le droit d'auteur. L'utilisation des services d'Érudit (y compris la reproduction) est assujettie à sa politique d'utilisation que vous pouvez consulter en ligne.

https://apropos.erudit.org/fr/usagers/politique-dutilisation/ 


\title{
La légende de Raphaël. Les «grandes» et les «petites» vies d'une figure exemplaire de l'art, écrites en France au cours du XIXe siècle
}

\author{
Nicole Dubreuil-Blondin, Université de Montréal
}

\begin{abstract}
$\longrightarrow$

onsidering the lives of Raphael written in nineteenth-century France, this article examines the epistemological status and the ideological function of an important genre of art historical writing: the artist monograph. During a period characterized by new scientific ambitions and by the development of the discipline within academic institutions, the Vasarian model of the vito undergoes a number of changes that do not, however, alter in a significant way its celebrational purpose. Especially throughout the biographical anecdotes that sustain the narrative of his famous career (like the episode of his love affair with la Fornarina), Raphael remains both the great man and the angelic figure concocted by tradition, combining pagan and
\end{abstract}

Christian standards of excellence, a pattern that finds itself repeated in the more popular versions of Raphael's lives, whether written for educational or for recreational purposes. The success story proposed by the life of Raphael - one of the artists most written about in France between 1800 and 1900 - establishes a poignant contrast with the destiny of the modern artist elaborated in contemporary literature, under the aegis of bohemia. It suggests that art history likes winners and not losers and that the genre of the artist monograph always serves some ideological purpose, like compensating fantasmatically for the economic, social and political insecurities of the times. e présent article, dont la longueur du titre rappelle les procédés d'énonciation à l'honneur au XIXe siècle, invoque le cas Raphaël pour aborder une question historiographique beaucoup plus actuelle: il s'agit en effet, à travers l'étude de monographies concernant l'artiste, d'amorcer un examen critique des procédés de l'histoire de l'art, au moment où la discipline se constitue en tant que pratique autonome se réclamant d'une forme d'objectivité "scientifique». Il ne s'agira donc pas ici d'isoler une tranche de la fortune critique de Raphaël, afin d'évaluer son apport déjà ancien au scholarship de l'artiste ou sa contribution à la connaissance générale de l'art italien. Pour le spécialiste travaillant à la poursuite de la vérité historique, le rétablissement des faits constitue une sorte d'opération autosuffisante et cumulative qui n'a pas besoin de traîner derrière elle une lourde archéologie. Aujourd'hui, les mérites du XIXe siècle dans la constitution du catalogue des oeuvres, de même que sa fascination pour la chronologie et les typologies stylistiques, retiennent peu l'attention. Ce qui est acquis est acquis; seuls les points encore obscurs méritent élaboration et les erreurs éventuelles, rectification. En revanche, surtout lorsqu'il s'agit d'une des figures exemplaires de l'histoire de l'art comme Raphaël, cette typologie a beaucoup à nous apprendre sur les conduites de notre discipline et sur l'idéologie informant ses présupposés théoriques et méthodologiques dans la mise en représentation du passé artistique.

La participation française du XIXe siècle à la constitution du dossier Raphaël prend ici toute sa pertinence. On pourrait s'étonner, de prime abord, de ce privilège accordé à une démarche qui s'est souvent affichée comme incorrigiblement littéraire et que la tradition savante germanique et anglo-saxonne a parfois tendance à soupçonner de super- ficialité. C'est cette tradition non française, en effet, qui nous a laissé les noms de Passavant ${ }^{1}$ de même que ceux de Crowe et Cavalcaselle ${ }^{2}$, à titre de références incontournables à la connaissance du peintre. Si l'on en croit Charles Clément $^{3}$, le contemporain de ces auteurs et l'un des contributeurs reconnus à l'exégèse de Raphaël, la France n'était toutefois pas en reste et procédait, pour ce qui concerne le maître d'Urbin, à une intense activité de rattrapage: "Les études sérieuses sur l'histoire des beaux-arts semblent, depuis quelques années, reprendre faveur en France, et notre littérature tend à conquérir le rang qu'elle doit y occuper, en suivant une voie où nous nous étions laissés devancer par l'Angleterre, par l'Italie, et surtout par l'Allemagne. C'est par Raphaël que l'on a commencé, et il faut espérer que les autres maîtres de l'art moderne ne tarderont pas à avoir leur tour..1 ${ }^{4}$ De Quatremère de Quincy ${ }^{5}$ à Eugène Müntz ${ }^{6}$, la liste est longue de ces auteurs, conservateurs de musées, titulaires de chaires d'enseignement, érudits de profession ou dilettantes avisés, à avoir consacré leurs meilleurs efforts au célèbre peintre italien. Les publications des Bigot ${ }^{7}$, Gruyer ${ }^{8}$, Rio9 ${ }^{9}$ et Vitet ${ }^{10}$ contribuent, avec beaucoup d'autres, à maintenir la figure de Raphaël au premier plan des artistes intéressant l'histoire de l'art française au XIXe siècle.

Cette frénésie d'érudition s'explique sans doute par l'impulsion historienne animant les Français au sortir de leur grande Révolution, une impulsion qu'est venue soutenir la montée du positivisme et qui a permis à la discipline de l'histoire d'usurper la place conventionnellement occupée par la philosophie dans la tradition universitaire ${ }^{11}$. Du côté de l'histoire de l'art, les contours de ce champ scientifique demeurent cependant un peu flous, témoignant d'une 
professionnalisation encore mal assurée et relevant d'institutions et de pratiques à vocations diverses, dont celle du journalisme ou de la littérature d'art qui continuent d'exercer un attrait considérable sur l'élite cultivée et sur le public en général. Une figure universellement célébrée comme celle de Raphaël attire donc, en plus des spécialistes reconnus, des auteurs de tout acabit et il n'est pas toujours facile de départager les véritables producteurs du savoir de ces diffuseurs de haut niveau, rédacteurs de synthèses sérieuses qui redisent et résument, en y glissant leurs propres considérations, les derniers acquis de la tradition savante. Au sein de ce groupe, pour ce qui concerne Raphaël, il faut inclure Ernest Breton ${ }^{12}$ de même que Charles Blanc, dont la célèbre Histoire des peintres de toutes les écoles constitue à la fois le classique et la somme du genre ${ }^{13}$. La dose d'érudition, dans ces écrits, se ramène habituellement à une question de format, les essais les plus brefs se contentant d'aller aux traits essentiels. L'effort littéraire, par contre, se dément rarement tout au long de la chaîne de ces textes, dans lesquels faire de l'histoire de l'art, c'est aussi raconter et décrire. La perspective historiographique qui est la nôtre nous interdit alors de négliger un vaste corpus d'écrits divers qui n'apportent pas nécessairement des éléments nouveaux à la connaissance de Raphaël. Nous les retenons pour deux raisons. L'opération de décantage qui s'y effectue permet en effer de dégager la doxa dans le foisonnement des détails, c'est-à-dire qu'elle facilite la mise à jour des éléments récurrents - sortes de lieux communs rarement conscients et peu remis en cause - qui déterminent le type de représentation artistique correspondant à une époque donnée (c'est là, est-il besoin de le souligner, que l'idéologie fait d'abord son lit!). L'autre avantage de ce vaste éventail d'écrits pour l'historiographe est d'attirer l'attention sur la pratique de l'histoire l'art comme discours, c'est-à-dire comme stratégie textuelle dont la structure même produit ses propres effets de sens et transmet tout un système de valeurs à considérer.

Si l'on excepte les études les plus pointues portant sur des fragments de l'oeuvre, sur des épisodes de la carrière ou sur des particularités du contexte, il est possible d'affirmer que la monographie, entendue ici dans sa formulation la plus conventionnelle où le récit de vie encadre celui du développement de l'art, constitue la grande catégorie d'écrits à laquelle se rattachent la plupart des textes concernant Raphaël produits au XIXe siècle. Selon Jacques Thuillier ${ }^{14}$, cette fascination pour le destin de Raphaël se trouve liée, en France, au destin même du genre puisque la vie de Raphaël par Vasari, dans la traduction qu'en a donnée Pierre Daret en 1651, constitue la première vie d'artiste publiée en sol français. La référence à la source vasarienne, une source extrêmement présente, nous le verrons, dans les écrits sur Raphaël faisant l'objet de notre analyse, permet déjà de rappeler à quel point la monographie d'artiste correspond à un genre idéologiquement marqué à cause de la conception de l'histoire qui la fonde. Remise à la mode du jour, suivant des modèles empruntés à l'Antiquité, dans les cercles humanistes que Raphaël a lui-même côtoyés, la monographie - on a encore trop tendance à l'oublier - est tout entière vouée à la glorification du grand homme, à ses actions d'éclat et à son immortelle postérité. On sait comment l'entreprise des Vite de Vasari ${ }^{15}$, en qui Germain Bazin $^{16}$, après beaucoup d'autres, reconnaît le père fondateur de notre discipline, s'est développée comme une sorte de greffe à ce véritable Panthéon d'hommes célèbres que constituait le Musaeum de Paolo Giovio. Cette galerie de portraits, authentiques ou fictifs, flanqués de petits abrégés de vies conçus sous formes d'elogia, offre déjà en effet la matrice structurelle des Vite et son caractère fondamentalement apologétique. Nous connaissons bien aujourd'hui à quels enjeux sociaux correspondait cette première littérature monographique développée par la Renaissance et tout entière vouée à la promotion de l'art au rang de pratique libérale. Dans l'optique de cette campagne publicitaire, où la réalisation d'une grande oeuvre vient remplacer l'action d'éclat, l'histoire se déploie à la faveur d'une intentionnalité souveraine capable d'en infléchir le cours.

Ainsi en va-t-il de la vie de Raphaël par Vasari. Alors qu'il se targue d'avoir eu un grand souci des faits et d'avoir sillonné l'Italie à la poursuite des oeuvres, des documents et des témoins lui permettant de retracer le Raphaël historique, Vasari n'en émaille pas moins son récit de toute une suite de topiques qui ont peu à voir avec l'enquête savante. Kris et $\mathrm{Kurz}^{17}$ nous ont montré qu'elles correspondent à des formes d'archétypes remontant à l'Antiquité et dont l'ultime origine se perd dans le mythe. Ces topoi, que viennent à peine camoufler les particularismes d'époque, se manifestent systématiquement dans les éléments biographiques proprement dits, c'est-à-dire dans ces anecdotes qui donnent au texte son cadre et sa charpente narrative. Si elles sont relativement peu nombreuses chez l'auteur italien, dont le premier souci demeure la présentation et la louange de l'oeuvre, elles n'en projettent pas moins autour de la figure de l'artiste l'aura du génie. Depuis l'enfance privilégiée par le destin (Raphaël, en opposition à la pratique la plus répandue dans son milieu, est nourri au sein maternel et élevé dans la maison de son père) et la révélation précoce du talent (Sanzio amène son fils au Pérugin qui ne peut que s'incliner devant les dons exceptionnels dont fait preuve son élève), la carrière de l'artiste correspond à une lente montée vers la gloire que vient sanctionner le deuil général en- 
tourant sa mort (l'épitaphe de Bembo, toute imprégnée de culture latine, reconnaît qu'il était d'une stature artistique à susciter la jalousie des dieux). En filigrane de ce récit, et conformément au synchrétisme propre à l'esprit renaissant, se dessine une version plus chrérienne ${ }^{18}$ (christique, même, par certains aspects) de cette figure héroïque dont la trajectoire est jalonnée de marques d'élection céleste. Né et mort un vendredi saint, dans la trentaine, ce peintre au nom angélique jouit des plus grandes faveurs divines si l'on en juge par certaines circonstances miraculeuses entourant sa vie et son oeuvre. Vasari rapporte qu'un de ses tableaux, le Spasimo, en route vers Palerme, s'est trouvé providentiellement sauvé d'un naufrage. La dernière image que l'auteur italien nous laisse de Raphaël, la tombée de rideau de cette représentation exemplaire, nous montre l'artiste étendu au pied de sa Transfiguration, l'ultime réalisation devant lui assurer symboliquement le salut éternel en plus de la renommée terrestre. Le témoignage des documents et le traitement de certains éléments biographiques s'accordent, dans le texte vasarien, avec une autre topique que Kris et Kurz, avant Wittkower, signalent comme fondamentale à la monographie d'artiste: il s'agit de l'identification de l'homme et de l'oeuvre, les traits de l'un passant subrepticement à l'autre et inversement. Ainsi voit-on se fabriquer, sous le double label de l'excellence païenne et chrétienne, le grand et le vertueux Raphaël qui traverse la vie en collectionnant les témoignages d'amitié, de reconnaissance et de vénération. Il existe bien quelques petites ombres au tableau, mais elles n'obscurcissent pas de manière significative le nimbe dans lequel la figure de Raphaël se trouve elle-même transfigurée. Il y aurait eu par exemple cette ambition mêlée de calcul qui lui aurait fait différer un mariage avantageux ${ }^{19}$ pouvant le priver d'un chapeau cardinalice; et puis il faudrait compter avec cette fièvre pernicieuse qui se mit à dévorer l'artiste jusqu'à causer directement sa mort. Le biographe semble l'imputer à la maîtresse qui vivait dans sa maison, dont il avait exécuté deux portraits, et qu'il fit partir avant de rendre l'âme, après lui avoir donné de quoi vivre honnêtement. Il aura donc suffi d'un testament, d'une ulcime confession du mourant, pour que le Raphaël de Vasari puisse passer au tribunal de la renommée avec un dossier expurgé de ses éléments les plus incriminants.

À plus de trois cents ans de distance, les auteurs reprenant le projet d'écrire la monographie de Raphaël semblent avoir troqué la toge ${ }^{20}$ pour la redingote noire de l'esprit moderne, à entendre Jacques Thuillier caractériser la démarche du XIXe siècle comme une tentative de rendre enfin le génie à l'histoire ${ }^{21}$. Le virage est d'autant plus marqué que les XVIIe et XVIIIe siècles français avaient eu tendance à absorber l'artiste dans de pures querelles d'esthétique, en l'impliquant, par exemple, dans la célèbre dispute opposant les poussinistes aux rubinistes ${ }^{22}$. Un événement symptomatique, parce qu'il nous ramène, justement, au tombeau de Raphaël, nous laisserait supposer que l'historiographie de l'après-Révolution s'établit sur de tout autres bases. En 1833, une lettre adressée à Quatremère de Quincy ${ }^{23}$ par un des membres de la commission chargée d'exhumer les restes de l'artiste (dans l'ancienne église Sainte-Marie de la Rotonde) en dit long sur le changement d'attitude qui anime la période: les proportions du squelette, l'état de conservation de la tête et des dents, le creux marqué par l'apophyse du bras droit à la suite de ce qui paraît avoir été un constant exercice manuel, font l'objet d'un examen minutieux et d'un rapport détaillé. Il s'avère alors que le grand homme, sous l'oeil scrutateur de la science, paraît simplement avoir été "...un individu de sexe masculin de petites proportions." Et quoique relativement intact, le corps (contrairement à celui du miraculé Spasimo), n’a pas échappé aux injures de l'eau, comme le révèlent les traces du limon du Tibre préservées dans la caisse. Quant au discours concernant l'artiste, si l'on en croit le commentaire d'Alfred de Lostalot sur la vie de Raphaël écrite par Eugène Müntz, il aurait lui aussi subi des rajustements: "Le temps n'est plus où l'on acceptait comme article de foi toutes les assertions de $\mathrm{Va}$ sari et des historiens qui l'ont répété: l'esprit scientifique du jour a des exigences auxquelles il faut absolument donner satisfaction.» ${ }^{24}$ Vasari demeure en effet la référence incontournable pour tous les auteurs du XIXe siècle, mais un Vasari que l'on traite comme un historien un peu négligent des dates, un peu sommaire dans ses appréciations et ses analyses et terriblement chauvin dans ses préférences. Cette période caractérisée par une intense activité muséale va d'abord renforcer ce qui, chez le biographe italien, constituait déjà un objectif majeur de la monographie d'artiste. Il s'agit de la connaissance de l'oeuvre pour laquelle il y a encore bien des pièces manquantes, notamment du côté des dessins, que les auteurs du XIXe siècle n'en finissent plus de découvrir en sillonnant les collections européennes. La production de cet artiste de grands chantiers, rompu au travail de collaboration en atelier et confiant de plus en plus les tâches d'exécution à des subalternes, pose aussi d'urgents problèmes d'attribution que vient compliquer le phénomène de la copie particulièrement répandu en ce qui concerne les tableaux de Raphaël. Il en découle une caractérisation de plus en plus fine du développement stylistique (ce que l'on appelle les "manières" de Raphaël) accompagnée d'un examen méticuleux des sources et des influences. Une attention considérable se trouve ainsi accordée aux débuts de la carrière et aux traditions provinciales qui ont présidé à son éclosion, depuis l'impulsion initiale donnée par le père 
artiste, Giovanni Santi. Le XIXe siècle français voit sans doute là l'occasion d'activer les grands principes de causalité que va si bien exposer la philosophie d'Hippolyte Taine, et de présenter l'homme comme le produit de sa race, de son milieu et de son moment ${ }^{25}$. Avec les études sur la religiosité de l'école ombrienne, le raffinement intellectuel de la Cour d'Urbin et la magnificence un peu trouble des cercles pontificaux, le décor et le destin de l'homme se précisent et s'ancrent dans une trajectoire historique aux multiples faisceaux: celui de la personne avec son hérédité et son entourage artistiques, celui de l'époque avec ses moeurs et ses traditions culturelles et celui de l'art italien, avec ses écoles marquées par des retards et des avancées stylistiques.

Les éléments biographiques n'ont pas été éliminés de tout cet effort. Dans ce domaine, cependant, peu de nouveaux documents voient le jour, peu de faits qui n'aient déjà été rapportés par Vasari ou par des sources italiennes anciennes, comme cette imputation de la mort de l'artiste à un refroidissement (plutôt qu'à quelque désordre amoureux) dont l'argument circulait depuis longtemps. La légende, par contre, s'est quelque peu rallongée au fil des époques, notamment en ce qui concerne les amours avec la Fornarina, cette maîtresse dont le XVIIIe siècle semble avoir suggéré le nom et amplifié quelque peu l'histoire. Que faire avec cet héritage relativement mince alors que la curiosité ne s'est aucunement atténuée, si l'on en croit le témoignage de Delacroix dans une notice consacrée justement à Raphaël: "Il ne nous reste sur la vie des grands maîtres que peu de renseignements auxquels l'histoire puisse se fier. Il est fâcheux que nous soyons si mal servis dans le désir naturel de nous instruire de ce qu'ils ont été, de la vie qu'ils ont menée...nous voudrions faire connaissance avec leurs personnes, bien plus, avec leurs bizarreries et leurs passions. Nous aimerions au moins à les trouver des hommes comme nous, dans la partie la plus vulgaire de la vie...n ${ }^{26}$ On imagine ici que cette aspiration, trahissant une certaine exaltation romantique mêlée à une bonne dose de prosaïsme bourgeois, puisse annoncer une remise en cause radicale du modèle vasarien et humaniste de la vie du grand homme ${ }^{27}$. Les choses ne sont pas aussi simples, cependant. Si le XIXe siècle révise ponctuellement certains épisodes biographiques, on doit reconnaître qu'il laisse intactes et la structure, et la visée ultimement célébrationnelle du modèle d'origine. Des efforts se manifestent, bien sûr, dans les textes les plus sérieux, pour dégager la vie de la fable et de l'hagiographie. Des dates se rectifient par un réexamen des documents: ainsi apprend-on qu'une concordance de quantièmes (7 avril), et non de jours fériés (Vendredi Saint), unit le moment de la naissance et le moment de la mort; que Raphaël a perdu ses parents jeunes et que c'est son oncle maternel, plutôt que son père, Santi, qui l'a mené chez le Pérugin; qu'il a aimé, puisqu'il a écrit des poèmes amoureux ponctués d'allusions personnelles; qu'il avait une maîtresse dont fait foi le testament (une Margarita et non une Fornarina, selon une annotation manuscrite retrouvée dans une ancienne édition de Vasari qui n'échappe pas aux nouvelles exigences de l'érudition); qu'il nous reste d'elle deux portraits vraisemblablement authentiques et des traces probables de sa physionomie dans les têtes de quelques madones de la période romaine; enfin que l'artiste a dû mourir d'avoir trop travaillé, à cause d'une complexion délicate facile à reconnaître dans ses autoportraits (l'observation clinique venant ici exorciser l'hypothèse du désordre amoureux). Qu'ils soient contestés ou simplement repris de Vasari et annoncés comme tels, sans autre forme de commentaire, comme ce sauvetage miraculeux du Spasimo apparaissant dans un bon nombre d'écrits, les éléments biographiques ne sont pas retenus dans le seul but de satisfaire à la vérité historique ou à la pure curiosité. Ils s'avèrent à la fois essentiels au récit qu'ils ponctuent et qu'ils relancent (une tranche de vie préparant une tranche de l'oeuvre et lui servant en quelque sorte de caution) et à l'édification d'une figure d'artiste qui continue d'apparaître en tous points exemplaire.

Dans les textes d'histoire de l'art du XIXe siècle, $\mathrm{Ra}$ phaël demeure en effet l'incarnation vivante de la perfection. Même expurgée de quelques fables, la trajectoire biographique se développe comme une forme de success story intégrale ne pouvant trouver sa finalité que dans l'apothéose. Au-delà des qualificatifs louangeurs et des superlatifs qui s'attachent à sa personne (les termes "génial» et "divin" reviennent à satiété, cautionnant l'apparition en chaîne de "chefs-d'oeuvre"), et dans lesquels persiste un fort parfum de l'antique fama, on peut dire que le Raphaël mis en scène par l'historiographie moderne n'a pas encore été arraché à son panthéon. Rappelons par exemple qu'un bon nombre de monographies de l'artiste apparaissent dans des volumes ou dans des séries traitant des grands maîtres du passé, c'està-dire baignant d'entrée de jeu dans une aura de gloire et de renommée. On semble privilégier aussi les études comparées qui regroupent Léonard, Michel-Ange et Raphaël, et surtout les deux derniers ${ }^{28}$, dont les personnalités contrastées représentent les voies complémentaires de l'excellence, tout en satisfaisant à un procédé d'exposition très recherché en histoire de l'art (le XIXe siècle ne connaît plus les dilemmes de Vasari, un allié historique de Michel-Ange, dans l'évaluation de ces météores de l'art qu'il fait régner côte à côte sur les cimes de la perfection). Quant au récit même de la vie de Raphaël, il paraît posséder tous les ingrédients facilitant la combinaison du donné historique 
objectif avec l'idéalité du modèle; la trajectoire du peintre, en effet, s'y présente systématiquement comme une montée vers l'accomplissement et la célébrité, depuis la provinciale Pérouse, où s'amorce son apprentissage, jusqu'à la Rome papale qui consacre son autorité et son triomphe. Les contacts artistiques de Raphaël et les milieux sociaux qu'il fréquente permettent à l'historien de déployer toute la panoplie des grands noms constituant l'élite civile, religieuse et culturelle de l'époque en même temps qu'elle lui fait franchir à l'accéléré les principales étapes du développement de l'art italien à un moment crucial pour l'histoire de l'art d'Occident. De Pérouse à Florence, et de Florence à Rome s'effectue le passage d'un monde encore gothique et religieux à cet espèce de plateau élevé que constitue encore, pour le XIXe siècle, l'humanisme renaissant. Quelle chance qu'une fin prématurée, malgré son caractère tragique, ait simultanément sauvé l'homme de la flétrissure, et son art de la décadence. Raphaël meurt fort à propos au milieu des gloires de son temps après avoir planifié sa sépulture dans cette église de sa paroisse qui portait jadis un nom prémonitoire: le Panthéon!

Au-delà des attributs de la grandeur, le XIXe siècle semble déterminé à préserver, à amplifier même, les hautes qualités morales et les traits de caractère exceptionnels que Vasari avait déjà reconnus à son artiste, notamment ceux de la douceur, de la modestie et de la convivialité. "SaintRaphaël, priez pour nous", s'exclame un auteur de la Revue des Deux-Mondes ${ }^{29}$ après avoir énu méré les marques de prédilection céleste dont jouissait le peintre. Rien de plus révélateur de cette tentation hagiographique que l'argumentation du méticuleux Campori, un collaborateur de la Gazette des Beaux-Arts ${ }^{30}$, plongé dans les archives de Modène à la recherche de nouveaux documents concernant Raphaël. L'artiste se voit systématiquement dégagé de tout soupçon de blâme que pourrait susciter la mise à jour de certains faits troulants. Le peintre a-t-il différé jusqu'à l'absurde la réalisation d'un tableau promis au duc de Ferrare? C'est qu'il était à la fois trop bon pour refuser la commande et trop occupé pour s'en acquitter. Aurait-il participé à la réalisation de décors de scène pour ces amusements de la cour papale dont les moeurs n'offraient décidément rien de très religieux ni de très édifiant? Il n'a pas dû..., il n'a pas pu... ou bien cette collaboration constitue un élément tout à fait épisodique qui doit avoir laissé intacte sa vertu. Une lettre de Pandolfo Pico à la marquise de Mantoue suggèret-elle que Raphaël aurait manifesté de la jalousie envers un jeune élève de Michel-Ange. Improbabilité, de commenter Campori, qui fait d'autre part grand état de cet événement remarquable rapporté dans la même lettre: au moment de la mort de l'artiste, des fissures importantes sont apparues dans le palais du Vatican que Raphaël était en train de restaurer, forçant le Pape à quitter ses appartements. Peut-on imaginer plus colossal voile du temple exprimant, par ses déchirements mêmes, la douleur généralisée accompagnant la disparition de cet être parfait? C'est dans ce registre moral que se manifeste le plus fortement, nous semble-t-il, la nécessité d'identifier l'artiste et son oeuvre qui habite tout projet monographique en histoire de l'art. Le XIXe siècle vient ici donner à une vieille topique les accents de la piété bourgeoise et catholique. Le Raphaël du XIXe siècle français est d'abord et avant tout le peintre de madones et de tableaux de dévotion dont l'iconographie, depuis les prestigieux originaux du Louvre jusqu'à la reproduction ad nauseam du motif dans l'imagerie religieuse, projette la figure de l'innocence et de la suavité. Les Vierges sont Raphaël; plus juvéniles dans la première "manière», elles accèdent avec lui à la plénitude d'une belle maturité qui ne connaîtra pas la flétrissure. Ce port de tête gracieux, alangui, de Marie ne se retrouve-t-il pas aussi dans les autoportraits auxquels il confere une attendrissante fragilité?

Toute l'affaire de la Fornarina, ce sujet périlleux que les auteurs les plus spécialisés abordent avec une extrême réserve, se voit ainsi largement reformulée dans l'optique de l'oeuvre. Au moment où l'on cherche les traces iconographiques de son passage dans la vie du peintre, il paraît évident au plus grand nombre qu'un amour capable de produire cant de beauté n'est pas la marque d'une déchéance morale imputable à quelque corruption des moeurs. En s'élevant lui-même dans son art, dont l'idéalisme lui interdisait toute forme de complaisance, Raphaël n'a-t-il pas contribué à ennoblir et à grandir cette femme d'origine modeste (la fable la disait fille de boulanger) qu'un auteur croit avoir retrouvée inscrite au registre d'un couvent après la mort du peintre? On subodore quelques relents d'hypocrisie et de machisme bourgeois dans le traitement de ces rapports dissymétriques où les termes de maître et de mầtresse n'appellent jamais les mêmes connotations. Bourgeoise aussi, peut-être, cette insistance sur l'épuisement à la tâche comme cause éloignée de la maladie fatale contractée par Raphaël. Les monographies du XIXe siècle nous semblent mettre un accent particulier sur le thème du travail dans la carrière de l'artiste: travail méritoire, puisque les dons du génie n'ont atteint leur pleine mesure, chez lui, qu'après un long et pacient apprentissage; travail fructueux, puisqu'il apporte à Raphaël la renommée en même temps que la fortune; travail socialement intégré, puisque s'y manifeste un parfait accord entre toutes les parties de la chaîne de production, depuis le puissant mécène jusqu'au plus humble assistant d'atelier. Qu'on ait fait de simples traits de caractère comme la gentillesse et la douceur du peintre, et l'in- 
croyable capacité que Vasari lui reconnaissait déjà d'avoir vécu en harmonie avec son entourage, un tel objet d'émerveillement cache sans doute plus qu'une propension à l'hagiographie de la part des historiens du XIXe siècle. La perfection de Raphaël, c'est peut-être aussi la projection d'une harmonie sociale fantasmée qui vient faire oublier les dures réalités du temps.

Cette représentation de plus en plus moralisante rejoint un vaste public, dont celui de la jeunesse chrétienne, par une littérature d'art édulcorée se développant en marge de la production savante, et proposant à l'imitation des masses les vies édifiantes des grands maîtres du passé. Dans son ouvrage consacré aux "Princes de l'art" ${ }^{31}$ et soumis à l'imprimatur des évêques, Mme Céline Fallet, qui dut être professeur ou maître de pension, insiste sur la sagesse et sur la modestie du petit Raphaël respectueusement soumis à l'enseignement de ses maîtres, un Raphaël besogneux que la Providence finit, bien sûr, par récompenser de ses largesses. Voilà ce que nous appellerions non pas une vie brève, mais plutôt, avec toutes les connotations péjoratives du terme, une "petite" vie de Raphaël dont l'apparition pourrait signaler une certaine crise du genre et un épuisement du modèle. À ranger du côté des "petites" vies, encore, ces productions romanesques et théâtrales qui donnent de l'artiste une image sentimentale ou vaudevillesque, en ramenant sa carrière à une suite d'intrigues amoureuses. Une remarque de Vasari sur la présence de la maîtresse du peintre à la Farnésine, sert de prétexte à Joseph Méry ${ }^{32}$, homme de lettres caustique et mondain, pour gloser en alexandrins et en prose sur la liaison de Raphaël et de la Fornarina. Dans un climat de complots où la quête du modèle idéal se ramène à la poursuite d'une aguichante blonde aux yeux bleus, le Raphaël de Méry, s'il manifeste plus de passion égoïste que de désintéressement artistique, n'en incarne pas moins, à sa manière, une autre figure de la réussite. Aimé des femmes, dont l'une irait jusqu'à tuer pour lui, il reste d'abord et avant tout l'enfant gâté des riches et des puissants qui l'aident à satisfaire ses moindres fantaisies. Même la romance n'a pas eu ici raison de cette vieille topique qui place l'artiste à l'égal des plus grands et qui lui vaut toutes les marques de considération sociale.

Quel contraste entre ces histoires à succès, qu'elles soient animées de prétentions scientifiques, de zèle religieux et pédagogique ou qu'elles correspondent à de pures fins de divertissement, et ce contre-récit qui élabore, ailleurs, du côté de la vie de bohème ${ }^{33}$, une autre représentation du destin de l'artiste au XIXe siècle. Celui-ci, qui possède la misère en partage, s'épuise en attendant une gloire qui tarde tant à venir qu'elle risque à tout moment de le faire sombrer dans le désespoir et dans la folie. Dépendant financiè- rement du bourgeois qu'il méprise et de l'usurier qui l'exploite, il a pour maîtresse une Mimi Pinson courant comme lui après la fortune et qui l'abandonnera d'un coeur léger au gré de sa fantaisie et de ses propres ambitions; à moins qu'ils ne s'éteignent l'un et l'autre prématurément, victimes d'un même mal social. Son activité semble une suite de dépenses improductives qui mènent rarement à l'achèvement et à la perfection de l'oeuvre. Sa trajectoire? Elle ne conduit pas de la maison du père à la maison du Pape, suivant ce vecteur ascendant qui résume la carrière de Raphaël. Il s'agit bien plutôt d'une dérive, que vient baliser une sorte de topographie de l'errance parisienne: de quartier en quartier, de café en café, de taudis en taudis. Ce type d'individu, d'individu moderne, n'a pas de toute évidence d'emprise sur l'histoire. C'est pourquoi l'histoire de l'art l'ignore et l'abandonne, pour l'instant du moins, à la littérature; car l'histoire de l'art n'aime pas les perdants!

\section{Notes}

1 La monographie de J. D. Passavant (Rafaël von Urbino und sein Vater Giovanni Santi, Leipzig, 1839) fut traduite à Paris, en 1860 , chez l'éditeur Vve Jules Renouard, dans une version corrigée et augmentée par l'auteur, avec des annotations de Paul Lacroix.

2 J. A. Crowe et G. B. Cavalcaselle, Raphaël, His Life and Works, with particular reference to recently discovered records and exhaustive study of extant drawings and pictures, Londres, J. Murray, I, 1882; II, 1885.

3 Charles Clément a entre autres publié: Michel-Ange, Léonard de Vinci, Raphaël, avec une étude sur l'art en Italie avant le XVIe siècle, et des catalogues raisonnés, historiques et biographiques, $\mathrm{Pa}$ ris, Michel Lévy frères, 1861.

4 Clément, "Raphaël», Revue des Deux Mondes, ler juin 1860, p. 670.

5 A. C. Quatremère de Quincy, L'Histoire de la vie et des ouvrages de Raphaël, Paris, Librairie de Charles Gosselin, 1824.

6 Eugène Müntz, Raphaël, sa vie, son oeuvre, son temps, Paris, 1881.

7 Charles Bigot, "Les fresques de Raphaël à la Farnésine", Gazette des Beaux-Arts, ler déc. 1882, p. 465-85; ler jan. 1883, p. 35 51; ler avril 1883, p. 281-95; ler déc. 1883, p. 472-76.

8 F. A. Gruyer, Raphaël et l'Antiquité, Paris, 1864; Les Vierges de Raphaël et l'iconographie de la Vierge, Paris, 1869; Les Fresques de Raphaël provenant de la Magliana, Paris, 1873; Raphaël peintre de portraits; fragments d'histoire et d'iconographie sur les personnages représentés dans les portraits de Raphaël, Paris, 1881.

9 Alexis-François Rio, Michel-Ange et Raphaël, avec un supplément sur la décadence de l'École romaine, Paris, Hachetre, 1867

10 Ludovic Vitet, "La fresque de S. Onoforio", Revue des Deux Mondes, 15 nov. 1850, p. 581-616; "La Madone de Pérouse de Raphaël au Louvre", Revue des Deux Mondes, 1 er mars 1870, p. 238-45; "La fresque de la Magliana”, Revue des Deux Mondes, ler mai 1873, p. 196-211. 
11 Voir à ce sujet W.R. Keylor, Academy and Community: The Foundation of the French Historical Profession, Cambridge, Harvard University Press, 1975.

12 Ernest Breton, Notice sur la vie et les ouvrages de Raphaël, SaintGermain, Imprimerie de Tournon, 1863.

13 Le tome concernant les écoles ombrienne et romaine est paru en 1870 .

14 Jacques Thuillier, "Raphaël et la France: Présence d'un peintre", dans Raphaël et l'art français, Paris, Éditions de la Réunion des musées nationaux, 1983.

15 Giorgio Vasari, Le Vite dé piu eccellenti Architetti, Pittori e Scultori Italiani da Cimabue insino à tempi nostri. Florence, 1550, 1568. Nous utilisons ici la traduction française réalisée sous la direction d'André Chastel, Paris, Berger Levrault, 1983.

16 Voir à ce sujet G. Bazin, Histoire de l'histoire de l'art de Vasari à nos jours, Paris, Albin Michel, 1986, p. 38-51.

17 Ernst Kris et Otto Kurz, Die Legende vom Künstler: Ein historischer Versuch, Vienne, 1934.

18 Il faudrait explorer plus longuement le vaste champ analogique qui se déploie ici et qui associe tour à tour Raphaël à une figure de Christ, d'ange ou de saint.

19 Avec la nièce du cardinal Bibiena.

20 Selon l'expression de Julius von Schlosser rapportée par Bazin, op. cit., p. 46.

21 Thuillier, op. cit., p. 25.

22 Ibid., p. 18-24. Voir aussi Vincenzo Golzio, The Complete Works of Raphaël, New York, Harrison House, 1969, p. 612-21.

23 Rapporté dans Quatremère de Quincy, op. cit., p. 461.

24 Alfred de Lostalot, "Raphaël. Sa vie, son oeuvre et son temps", Gazette des Beaux-Arts, ler déc. 1881, p. 551.
25 Hippolyte Taine, Essais de critique et d'histoire, Paris, Hachette, 1858; 2e édit. avec nouvelle préface, ibid., 1866; 10 e édit. définitive, ibid., 1904.

26 Eugène Delacroix, "Raphaël», L'Artiste, juin 1872, p. 13.

27 Voir, sur la fin du genre, Marc Fumaroli, "Des vies à la biographie: le crépuscule du Parnasse», Diogène, 139, juillet-sept. 1987. p. 3-31.

28 Citons, en plus du livre de Clément mentionné en note 4, Henry Axenfeld, Les grands peintres Léonard de Vinci, Michel-Ange et Raphaëh Paris, Librairie Lecène et Oudin, 1889; Emile Ollivier, Une visite à la chapelle des Médicis. Dialogue sur Michel-Ange et Raphaëh Paris, Sandoz et Fisbacher, 1872; Müntz, "Une rivalité d'artistes au XVle siècle, Michel-Ange et Raphaël à la cour de Rome", Gazette des Beaux-Arts, ler mars 1882, p. 281-87; ler avril 1882, p. 385-400.

29 Henri Blaze de Bury, "Raphaël et Michel-Ange. Leur vie mondaine et politique. Leurs poésies et leurs amours", Revue des Deux Mondes, ler fév. 1878, p. 484.

30 Giovanni Campori, "Documents inédits sur Raphaël tiré des Archives palatines de Modène", Gazette des Beaux-Arts, ler avril 1863, p. 347-61; ler mai 1863, p. 442-56; ler sept. 1863, p. 288-94.

31 Céline Fallet, Les Princes de l'art. Architectes, sculpteurs, peintres, graveurs, musiciens, poètes, orateurs, Rouen, 1859, 1862, 1863, $1865,1866,1868,1873$.

32 Joseph Méry, Raphaël. Comédie historique, en 3 actes, en vers, Paris, Librairie de Paul Dupont, 1851; Raphaël et la Fornarine. Roman inédit, Paris, Gabriel de Gonet, 1854.

33 Nous nous référons, entre autres, à Henri Mürger, Scènes de la vie de Bohème, 1851; Champfleury, Chien-Caillou, 1857; Edmond et Jules de Goncourt, Manette Salomon, 1867. 